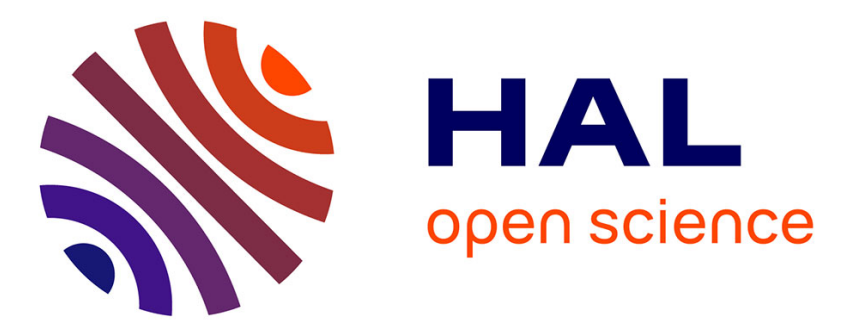

\title{
In situ assessment of the contact angles of nanoparticles adsorbed at fluid interfaces by multiple angle of incidence ellipsometry
}

Antonio Stocco, Ge Su, Maurizio Nobili, Martin In, Dayang Wang

\section{- To cite this version:}

Antonio Stocco, Ge Su, Maurizio Nobili, Martin In, Dayang Wang. In situ assessment of the contact angles of nanoparticles adsorbed at fluid interfaces by multiple angle of incidence ellipsometry. Soft Matter, 2014, 10 (36), pp.6999. 10.1039/C4SM00482E . hal-01064084

\section{HAL Id: hal-01064084 \\ https://hal.science/hal-01064084}

Submitted on 15 Sep 2014

HAL is a multi-disciplinary open access archive for the deposit and dissemination of scientific research documents, whether they are published or not. The documents may come from teaching and research institutions in France or abroad, or from public or private research centers.
L'archive ouverte pluridisciplinaire HAL, est destinée au dépôt et à la diffusion de documents scientifiques de niveau recherche, publiés ou non, émanant des établissements d'enseignement et de recherche français ou étrangers, des laboratoires publics ou privés. 
Table of contents entry:

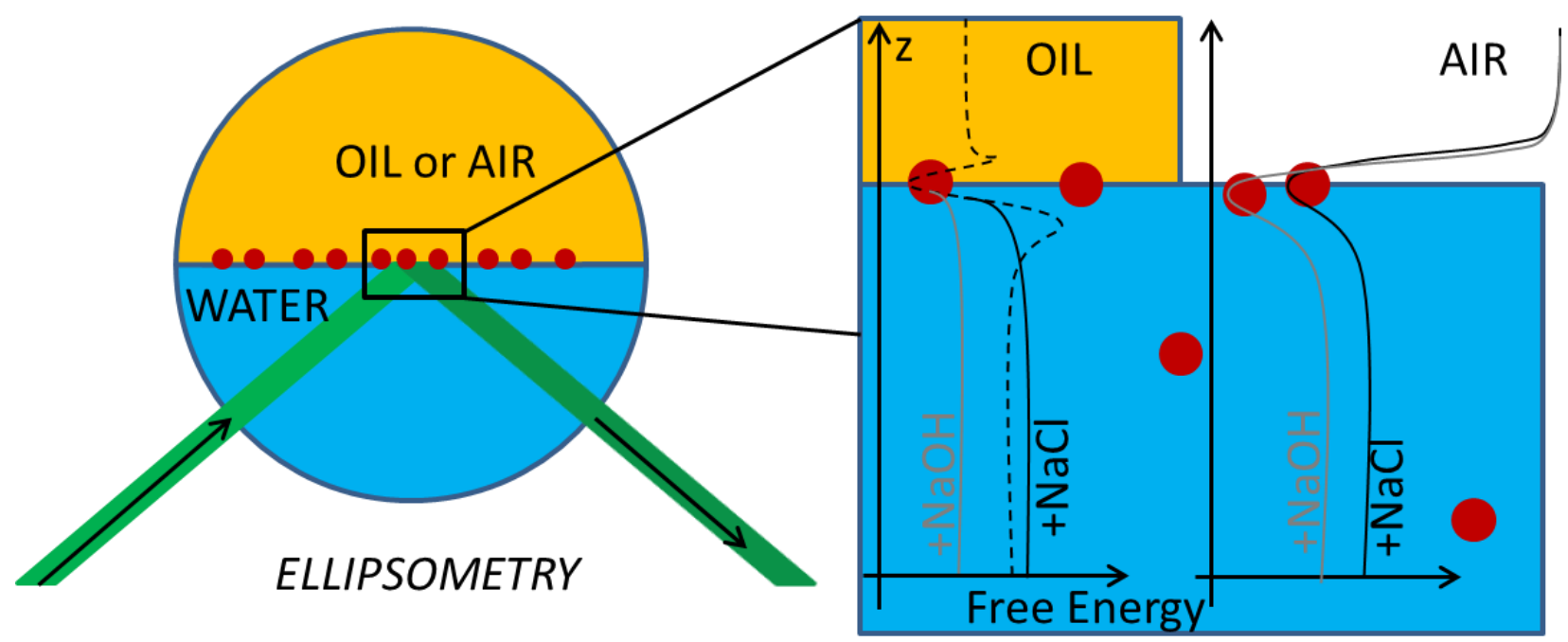

Contact angle and surface coverage of nanoparticles adsorbing at the fluid interface are assessed by ellipsometry. Results reveal the competition between wetting and colloidal interactions. 
TITLE: In situ assessment of the contact angles of nanoparticles adsorbed at fluid interfaces by multiple angle of incidence ellipsometry.

\author{
AUTHORS: Antonio Stocco* ${ }^{1,2}, \mathrm{Ge} \mathrm{Su}^{3}$, Maurizio Nobili ${ }^{1,2}$, Martin $\mathrm{In}^{1,2}$, Dayang Wang ${ }^{3}$ \\ 1) Université Montpellier 2, Laboratoire Charles Coulomb UMR 5221, F-34095, Montpellier, France \\ 2) CNRS, Laboratoire Charles Coulomb UMR 5221, F-34095, Montpellier, France \\ 3) Ian Wark Research Institute, University of South Australia, Adelaide, SA 5095, Australia.
}

\title{
*Antonio.Stocco@univ-montp2.fr
}

\begin{abstract}
Here multiple angle of incidence ellipsometry was successfully applied to in situ assess the contact angle and surface coverage of gold nanoparticles as small as $18 \mathrm{~nm}$, coated with stimuliresponsive polymers, at water-oil and water-air interfaces in the presence of $\mathrm{NaCl}$ and $\mathrm{NaOH}$, respectively. The interfacial adsorption of the nanoparticles was found very slow and took days to reach a fairly low surface coverage. For water-oil interfaces, in-situ nanoparticle contact angles agree with the macroscopic equilibrium contact angles of planar gold surfaces with the same polymer coatings; whilst for water-air interfaces, significant differences have been observed.
\end{abstract}

\section{Introduction}

When two immiscible fluids (gas or liquid) and a solid meet, the equilibrium of surface forces on the triple line leads to an equilibrium contact angle. The latter quantity is an important property of 
the solid, and its measurement is largely exploited for surface characterization. ${ }^{1}$ When the solid is a spherical particle straddling a fluid interface, an equilibrium contact angle can be also defined by the colloid immersion depth into one fluid (see Fig. 1D).

The measurement of the equilibrium contact angle of solid particles at the fluid interface is crucial to understand and predict dispersion, stabilization, phase transfer, and phase inversion in dispersed multiphase systems such as Pickering emulsions and particle stabilised foams. ${ }^{2}$ When particles possess sizes in the colloidal scale, equilibrium contact angle is determined not only by wetting but also by a competition of various colloidal interactions. ${ }^{3}$ Hence, the equilibrium contact angle of a colloidal particle can differ significantly from the macroscopic equilibrium contact angle measured by the far field view of a drop deposited on a support made of the same material.

Gehring and Fischer reported that the immersion of nanoparticles (NPs) at the water-air interface depend strongly on the ionic strength and the surface potential of water and the NPs. ${ }^{4,5}$ The contact angles of negatively charged NPs decrease with the ionic strength increasing, whereas positively charged NPs behave oppositely. ${ }^{6}$ In Pickering emulsions, it has been also reported that interfacial electrostatics strongly affects the interfacial curvature of the emulsion droplets, which is related to the partitioning of particles at the oil/water interface. ${ }^{7}$ For core-shell NPs, recent simulation studies suggest that the hydrophobicity of polymer shells strongly affects the position of the NPs relative to the interface. 8,9

Up to date, however, it remains an experimental challenge to in situ measure the location of colloidal particles at the fluid interface, particularly when the particle size is in the nanometric range and the liquid-liquid interface is considered. To overcome the experimental challenge, several indirect contact angle measurements were developed. ${ }^{10,11}$ New methods based on X-Ray 
or Neutron Reflectivity measurements have recently been proposed. ${ }^{12,13}$ Nonetheless, the most popular method to evaluate the contact angle of colloidal particles at the fluid interface is to measure macroscopic wetting contact angles (advancing and receding). It is also widely accepted that the equilibrium contact angle of an isolated colloid at the fluid interface is equivalent to the macroscopic equilibrium contact angle of model planar substrates. ${ }^{14,15}$ These two contact angles, however, differ in terms of length scales, interactions, and phenomena. ${ }^{3}$

Herein the contact angle and surface coverage of gold NPs at water-oil and water-air interfaces have been measured directly and in situ measured by ellipsometry. Multiple angle of incidence ellipsometry was already used to measure the contact angle and surface concentration of NPs at the liquid-gas interface. ${ }^{16,17}$ Here, we implemented a cylindrical sample holder into the experimental setup to investigate also liquid-liquid interfaces without the need of using optical waveguides. ${ }^{18}$ Multiple angle of incidence ellipsometry has been used to study NPs at watertoluene and water-air interfaces. This investigation aims at shedding light on the mechanism of NP adsorption to and especially desorption to the oil phase across the water-oil interfaces reported in recent publications. ${ }^{19,20}$ NP adsorption at water-air interfaces was also investigated as reference, in which no NP transfer to the air across the water-air interfaces could take place. The present work focused on the study of the effect of two different salts, $\mathrm{NaCl}$ and $\mathrm{NaOH}$, occurring at room temperature. $\mathrm{NaCl}$ is expected to increase the NP surface hydrophobicity and thus lower the interfacial potential barrier, which drives to NP transfer from water to toluene across the water-toluene interface. $\mathrm{NaOH}$ is expected to promote the surface hydration of NPs, thus suppressing the NP interfacial adsorption and crossing. ${ }^{20}$ As suggested in literature, it is worth noting that (i) no phase transfer was observed at room temperature in the absence of salt, and (ii) 
NPs transfer from water to toluene in presence of $\mathrm{NaCl}$ but do not transfer back to water when salty water is replaced by pure de-ionized water.

\section{Materials and Method}

\subsection{Gold NPs}

Citrate-stabilized gold NPs were coated with the chains of random copolymers of 2-(2methoxyethoxy)ethyl methacrylate $\left(\mathrm{MEO}_{2} \mathrm{MA}\right)$ and oligo(ethylene glycol) methyl methacrylate (OEGMA) via ligand exchange, as described in our previous reports. ${ }^{18}$ The polymers were synthesized via atom transfer radical polymerization using disulfide initiators and the polymer used in this work had a $\mathrm{MEO}_{2} \mathrm{MA}$-to-OEGMA molar ratio of 90:10. The number averaged hydrodynamic radius of naked gold NP cores $\left(\mathrm{R}_{1}\right)$ is $9.22 \mathrm{~nm}$ and the core-shell NP radius $\left(\mathrm{R}_{2}\right)$ is $13.81 \mathrm{~nm}$, measured by dynamic light scattering (see Fig. 1A, 1B). The refractive index of gold at the wavelength $\lambda=533 \mathrm{~nm}$ is $\mathrm{n}_{\mathrm{NP}}=0.63-2.30 \mathrm{i}$, and density $19.3 \mathrm{~g} \mathrm{~cm}^{-3} \cdot 21,22,23$

The water used is Milli-Q water. Toluene (Sigma-Aldrich), $\mathrm{NaCl}$ (Sigma-Aldrich) and $\mathrm{NaOH}$ (Sigma-Aldrich) were used as received.

\subsection{Sample preparation for ellipsometry measurements}

The sample holders used for ellipsometry were cylindrical glass cells $(7 \mathrm{~cm}$ diameter, $8.5 \mathrm{~cm}$ length, interface area $\mathrm{A}=59.5 \mathrm{~cm}^{2}$ and total Volume $208 \mathrm{~cm}^{3}$ ). The cells were half filled with water and half with either oil or air. The cells were precisely aligned in the center of the ellipsometer (Optrel, Germany) to ensure the normal incidence of the laser beam on the glass and thus to avoid deflation and reflection of the incident and reflected beams on the cell walls (see Fig. 1C). ${ }^{18}$ The interface level was adjusted to be in the correct vertical position, namely, at the center of the goniometer. 
$1 \mathrm{~mL}$ of the relatively concentrated aqueous dispersion of gold NPs was added to the cells to reach a NP concentration of $\mathrm{c}_{\mathrm{NP}}=1 \mathrm{gL}^{-1}$ in the water phase in the cells, corresponding to a volume fraction of $5 \cdot 10^{-5} \mathrm{~m}^{3} / \mathrm{m}^{3}$. Afterwards, the cells were shacked in order to quickly form homogenous NP dispersions. The filled cells were closed during experiments to avoid contamination of the interfaces. The ellipsometry measurements were carried out at room temperature $\mathrm{T}=22{ }^{\circ} \mathrm{C}$.

\subsection{Ellipsometry and modelling of NPs at interfaces}

Ellipsometry measures the ratio of reflection field coefficient $r_{P}$ (P-polarization, parallel to the reflection plane) and $r_{S}$ (S-polarisation, perpendicular to reflection plane). Amplitude $\tan \Psi$ and phase $\Delta$ changes can be accurately measured. The ellipsometry equation reads: ${ }^{24}$

$$
\frac{r_{P}}{r_{S}}=\tan \Psi \exp (i \Delta)
$$

where $r_{P} / r_{S}$ depends on the profile of the refractive index $\mathrm{n}(\mathrm{z})$ or dielectric constant $\varepsilon(\mathrm{z})=\mathrm{n}(\mathrm{z})^{2}$ across the interface, the bulk refractive indices $\left(\mathrm{n}_{\mathrm{i}}\right)$, the laser wavelength $(\lambda)$ and the angle of incidence $(\varphi)$.

For optically thin interfacial layers (whose thickness $<\lambda$ ), the perturbation theory ${ }^{25}$ describes $r_{P} / r_{S}$ as the sum of $r_{P, 0} / r_{S, 0}$ for the ideal step-like profile between the two bulk media and $J_{l}$, the term accounting for the first order deviation parameter:

$\frac{r_{P}}{r_{S}}=\frac{r_{P, 0}}{r_{S, 0}}-\frac{2 i Q_{1}}{r_{S, 0}\left(Q_{1}+Q_{2}\right)^{2}} \frac{K}{n_{0}^{2} n_{2}^{2}} J_{1}$

where $\mathrm{Q}_{1}=2 \pi /\left(\mathrm{n}_{1} \lambda\right) \cos \varphi, \mathrm{Q}_{2}=2 \pi /\left(\mathrm{n}_{2} \lambda\right) \cos \varphi_{2}\left(\varphi_{2}=\arcsin \left(\mathrm{n}_{1} \sin \varphi / \mathrm{n}_{2}\right)\right)$, and $\mathrm{K}=2 \pi \mathrm{n}_{1} / \lambda \sin \varphi$. For locally isotropic interfacial refractive index, $J_{l}$ is described as: 
$J_{1}=\int \frac{\left(\varepsilon(z)-\varepsilon_{1}\right)\left(\varepsilon_{2}-\varepsilon(z)\right)}{\varepsilon(z)} d z$,

Eq. 3

where $\varepsilon_{\mathrm{i}}=\mathrm{n}_{\mathrm{i}}^{2}$ and $\mathrm{z}$ is the axis normal to the interface (see Fig. 1D). In the limit of thin optical layers, ellipsometry data can be fitted with a single parameter $\left(J_{l}\right)$. Note that when the perturbation theory holds, $\tan \Psi$ is always minimum and $\Delta=90^{\circ}$ or $270^{\circ}$ at the Brewster angle $\varphi_{\mathrm{B}}$ $=\arctan \left(\mathrm{n}_{2} / \mathrm{n}_{1}\right)$.

For optically thick layers, the perturbation theory is no longer valid and Eq. 2 cannot describe multiple angle of incidence ellipsometric data. Recently, Hunter et al. ${ }^{16}$ and Zang et al. ${ }^{17}$ introduced a model able to fit data obtained for optically thick interfacial layers bearing NPs at the liquid-gas interface. Using the Maxwell-Garnett effective medium approximation, the interfacial profile of the dielectric constant $\varepsilon(\mathrm{z})$ is described as composed by a first layer accounting for the portion of NPs immersed in the first medium (e.g. water) and a second layer accounting for the portion of the NPs immersed in the second medium (e.g. oil or air). The model is schematically depicted in Fig. 1D. Knowing media dielectric constant $\varepsilon_{\mathrm{i}}$ and the particle radius $\mathrm{R}$ (which can be measured independently), the free parameters of the model are (i) $h$, the distance between the center of particles and the interface and (ii) $\phi$, the surface coverage described as $\phi=\mathrm{N} \pi \mathrm{R}^{2} / \mathrm{A}$, Eq. 4 where $\mathrm{N}$ is the number of particles in the interfacial region. Hence, the particle contact angle can be calculated as $\Theta=\arccos (-\mathrm{h} / \mathrm{R})$. In the model, the thickness of the first layer is $\mathrm{R}-\mathrm{h}$ and the one of the second layer is $\mathrm{R}+\mathrm{h}$. The dielectric constants of these two layers are calculated as below:

$\varepsilon_{L 1}=\varepsilon_{1} \frac{\varepsilon_{1}\left(2-2 f_{N P, 1}\right)+\varepsilon_{N P}\left(1+2 f_{N P, 1}\right)}{\varepsilon_{1}\left(2+f_{N P, 1}\right)+\varepsilon_{N P}\left(1-f_{N P, 1}\right)}$ 
$\varepsilon_{L 2}=\varepsilon_{2} \frac{\varepsilon_{2}\left(2-2 f_{N P, 2}\right)+\varepsilon_{N P}\left(1+2 f_{N P, 2}\right)}{\varepsilon_{2}\left(2+f_{N P, 2}\right)+\varepsilon_{N P}\left(1-f_{N P, 2}\right)}$

Eq. 6

where $\varepsilon_{1}$ and $\varepsilon_{2}$ are the dielectric constants of the first medium (i.e. water) and the second

medium (i.e. oil or air), respectively, $\varepsilon_{\mathrm{NP}}=\mathrm{n}_{\mathrm{NP}}{ }^{2}$ is the dielectric constant of NPs, $f_{N P, l}$ and $f_{N P, l}$ are the volume fractions of the portion of the NPs immersed in the layers 1 and 2, respectively:

$f_{N P, 1}=\phi \frac{2 / 3 R^{3}-\left(R^{2} h-h^{3} / 3\right)}{R^{2}(R-h)}, f_{N P, 2}=\phi \frac{2 / 3 R^{3}+\left(R^{2} h-h^{3} / 3\right)}{R^{2}(R+h)}$.

Eq. 7

\subsection{Macroscopic contact angle measurement}

Gold substrates were immersed overnight in $1 \mathrm{mg} / \mathrm{ml} \mathrm{MEO}_{2} \mathrm{MA}_{90}-\mathrm{Co}_{-} \mathrm{OEGMA}_{10}$ polymer solution, and then washed with water and dried under nitrogen gas stream. The contact angle was measured using the sessile method and captive bubble technique. Analysis was performed with an in-house software program. For sessile drop method, $2 \mu$ liquid was perpendicularly dropped onto polymer coated gold substrate. The contact angles, measured through the liquid phase, were captured and recorded by a progressive scan CCD camera (Dataphysics OCAH200). Static, advancing and receding contact angles $\left(\theta_{\mathrm{S}}, \theta_{\mathrm{A}}\right.$ and $\theta_{\mathrm{R}}$, respectively) were measured by the captive bubble method, in a quartz cell filled with water or electrolyte solution $(0.1 \mathrm{M} \mathrm{NaCl}$ or $0.1 \mathrm{M}$ $\mathrm{NaOH})$. A clean stainless steel needle was used to produce an air bubble or a drop of toluene of approximately $2 \mathrm{~mm}$ in diameter on the surface. The silhouette of the bubble/drop was captured. Advancing and receding contact angles, measured through air or toluene phase, were determined by setting a tangent to the contact line. Five measurements at different locations on each sample were made. All measurements were performed at least three times. 
From advancing and receding angles, equilibrium contact angles have been calculated in the framework of a model that incorporates the contact line energy into the Young-Laplace equation: $^{26}$

$\theta_{E}=\arccos \left(\frac{\Gamma_{A} \cos \theta_{A}+\Gamma_{R} \cos \theta_{R}}{\Gamma_{A}+\Gamma_{R}}\right)$

Eq. 8

where $\Gamma_{A}=\left(\frac{\sin ^{3} \theta_{A}}{2-3 \cos \theta_{A}+\cos ^{3} \theta_{A}}\right)^{1 / 3}, \Gamma_{R}=\left(\frac{\sin ^{3} \theta_{R}}{2-3 \cos \theta_{R}+\cos ^{3} \theta_{R}}\right)^{1 / 3}$.

\section{Results}

We started investigating the adsorption of gold nanoparticles at interfaces in the absence of salt in the water phase. Figure 2 shows the ellipsometry measurements of water-toluene and water-air interfaces in the absence and presence of gold NPs in the water phase. Before the NPs are introduced into the water, both water-toluene and water-air interfaces show the expected step-like change of $\Delta$ as a function of the incident angle $\varphi$ around the Brewster angle, which can be fitted well by the perturbation theory. $\mathrm{J}_{1}=0 \pm 0.1 \mathrm{~nm}$ (see Eq. 2 and 3 ) describes well the tiny deviation between the real dielectric constant profile across the interfaces and the abrupt step change between the dielectric constants of bulk phases. Surface roughness due to capillary waves and intrinsic density profile models have been used to explain the ellispometric parameter $\mathrm{J}_{1}{ }^{27,28}$

When the gold NPs are introduced into the water phase, they are expected to induce a high optical contrast and large change of the interfacial profile of the dielectric constant $\varepsilon(\mathrm{z})$ at watertoluene or water-air interfaces provided the NPs populate the interfaces. Note that the concentration of NPs used was $c_{\mathrm{NP}}=1 \mathrm{gL}^{-1}$ corresponding to a volume fraction of $5 \cdot 10^{-5}$, which was sufficiently low for the change of bulk refractive index to be negligible $n_{1}=\sqrt{\varepsilon_{1}}$, while 
sufficiently high to allow full coverage of the interfaces provided the NPs adsorbed to the interfaces. As shown in Figure 2, however, the changes of $\tan \Psi$ and $\Delta$ around the Brewster angle is negligible upon addition of gold NPs into water, which remain approximately the same as the interfaces before the NP addition. The Brewster angle does not shift. Water-toluene and water-air interfaces show the same results, indicating negligible adsorption of gold NPs from deionized water onto its surface.

\section{FIGURE 2}

The adsorption scenario completely changed when gold NPs were introduced in salty water phase. Figure 3a shows different ellipsometric scans measured for water-toluene interfaces and at different time $t$ elapsed after addition of gold NPs into water in the presence of $\mathrm{NaCl}(0.1 \mathrm{M})$. Comparing to the data shown in Fig.2, not only the slopes of $\tan \Psi$ and $\Delta$ change but, more importantly, the pseudo Brewster angle, defined as the angle where $\tan \Psi$ is minimum and $\Delta=$ $90^{\circ}$ or $270^{\circ}$, noticeably shifts with times. The shift of the pseudo Brewster angle is the sign of the NP adsorption onto the interface. The ellipsometric data hardly change after use of $0.1 \mathrm{M} \mathrm{NaOH}$ instead of $\mathrm{NaCl}$ in the water phase (Fig. 3b). Similar changes are also observed for gold NP adsorption onto water-air interfaces in presence of $\mathrm{NaCl}$ and $\mathrm{NaOH}$ (Fig 4). Note that for both water-toluene and water-air interfaces in the presence of $\mathrm{NaCl}$ or $\mathrm{NaOH}$ in water (Figs 3 and 4), $\tan \Psi$ and $\Delta$ change slowly with time upon addition of gold NPs; the kinetics time is of the order of days.

\section{FIGURE 3}

FIGURE 4 
The perturbation theory, valid for optically thin layers, cannot describe the data showing changes of the pseudo Brewster angle (see Eq. 2). Here we fitted all data using the model described in the previous section (Eqs. 4-7). In the present model, we used the bulk dielectric constants of gold, air, toluene and $\varepsilon_{\mathrm{i}}$ of the salty aqueous solutions from the bare interfaces data fitted using the perturbation theory. The polymer shells are in fact matching the dielectric constant of the oil phase; and the optical contrast of the polymer shell in water is negligible with respect to the optical contrast given by gold, so the radii of gold NP cores can be used to represent the radii of whole core-shell NPs, $R=R_{1}$. Thus, the only two fitting parameters are the surface coverage $\phi$ and the height $h$ (see Fig. 1). Figure 5 shows the evolution of the contact angle $\Theta=\arccos (-h / R)$ and $\phi$ with the NP adsorption time at water-toluene and water-air interfaces. For NP adsorption at water-toluene interfaces, $\Theta=91^{\circ} \pm 0.5^{\circ}$ and $\phi$ changes between 0.020 and 0.025 when $\mathrm{NaCl}$ is present in water. When $\mathrm{NaOH}$ is used instead of $\mathrm{NaCl}$ in water, $\Theta$ becomes slightly smaller $\left(90^{\circ} \pm 0.5^{\circ}\right)$, while $\phi$ is is significantly increased; 0.04 at $\mathrm{t}=6 \times 10^{5} \mathrm{~s}$.

For NP adsorption at water-air interfaces, as shown in Figure $6, \Theta=67^{\circ} \pm 4^{\circ}$ when $\mathrm{NaCl}$ is water and $\Theta=21^{\circ} \pm 12^{\circ}$ when $\mathrm{NaOH}$ is in water at $\mathrm{t}=6 \times 10^{5} \mathrm{~s}$. The surface coverage in both cases increases systematically from 0.024 to 0.055 with the NP adsorption time $(t)$. Note that at waterair interfaces, the optical contrast of the polymer shell in air is not negligible, so we explored the possibility of using a core-shell NP model to fit the data (see Appendix). To avoid a large number of free parameters, we assumed a spherical geometry of the core-shell particle defined by a core radius and a single core-shell radius. This assumption, however, cannot account for several polymer shell conformations: (i) the polymer could adopt a collapsed state in air and an extended 
state in water, or (ii) the polymer could prefer to remain flat at the interface. Indeed, recent simulations and experiments show that the polymer shell may prefer remaining in contact with the air-water interfaces in a pancake-like conformation. ${ }^{29,30}$ The latter considerations might be the reason why the core-shell NP model (see Appendix) is not suitable to fit the experimental data shown here. This model may work for core-shell NPs, which shells cannot adopt different conformation (e.g. silica-gold NPs). This work is underway.

\section{FIGURE 5}

For comparison, the macroscopic contact angles $\left(\theta_{S}, \theta_{A}\right.$ and $\left.\theta_{R}\right)$ were measured on planar gold substrates, coated with the brushes of random co-polymers of MEO2MA and OEGMA, which was implemented in the same way as the polymers were coated on gold NPs. Table 1 lists the macroscopic angles and equilibrium contact angles $\theta_{\mathrm{E}}$ calculated form advancing and receding angles (see Eq. 8). ${ }^{26}$ At the water-toluene interfaces $\theta_{\mathrm{E}}=93^{\circ}$ and it decreases slightly in presence of $0.1 \mathrm{M}$ of $\mathrm{NaCl}\left(\theta_{\mathrm{E}}=92^{\circ}\right)$ or $\mathrm{NaOH}\left(\theta_{\mathrm{E}}=87^{\circ}\right)$. For aqueous-air interfaces, no significant changes were observed when water is replaced by $0.1 \mathrm{M} \mathrm{NaCl}$ or $\mathrm{NaOH}$ solutions and $\theta_{\mathrm{E}}=53^{\circ}$.

\section{Discussion}

4.1 Adsorption. One of the main results of this experimental work is the effect of $\mathrm{NaCl}$ and $\mathrm{NaOH}$ on NP adsorption. Here, we clearly show that NPs do not accumulate and attach onto the pure water interfaces, being the ellipsometric measurements shown in Fig. 2 almost identical before and after the addition of NPs into water. A potential adsorption barrier as the double layer electrostatic repulsion exists close to interface on the water side, which hinder the NP 
adsorption. ${ }^{31}$ The screening of this repulsion is achieved by adding ionic species close to the interface. From this viewpoint, $\mathrm{NaCl}, \mathrm{NaOH}$ or other salts ${ }^{32}$ might lead to the same screening and consequently to NP adsorption. Moreover equilibrium or pseudo-equilibrium contact angles and surface coverages can be measured only at $t>1$ day, and the kinetic of adsorption is very slow when compared to the adsorption under diffusion control of nonionic surfactants. ${ }^{33}$

4.2 Surface Coverage. Now we can turn our attention to the surface coverages of NPs at salty interfaces shown in Fig. 5. For all interfaces, $\phi$ are much lower than the fully packed value of $\phi=\pi \mathrm{R}_{1}^{2} / 4 \times \mathrm{R}_{1}{ }^{2} \sin \left(60^{\circ}\right)=0.907$, calculated for hexagonally packed nanoparticles in contact by the inner cores; or the value of $\phi=\pi \mathrm{R}_{1}{ }^{2} / 4 \times \mathrm{R}_{2}{ }^{2} \sin \left(60^{\circ}\right)=0.404$, calculated for hexagonally packed nanoparticles in contact by the outer polymer shells (see Fig. 6A). Assuming a hexagonal lattice NP conformation at the interface, an average distance between particles at the interface can be estimated as $l=\sqrt{\frac{\pi R_{1}^{2}}{\phi \sin \left(60^{\circ}\right)}}$, which is $124 \mathrm{~nm}\left(l-2 \mathrm{R}_{2}=96 \mathrm{~nm}\right)$ for $\phi=0.020$ and $75 \mathrm{~nm}$ $\left(l-2 \mathrm{R}_{2}=47 \mathrm{~nm}\right)$ for $\phi=0.055$. One might discuss such $l$ values as due to long range repulsive interactions acting between adsorbed NPs. ${ }^{34}$ However, ellipsometry does not distinguish between different NP arrangements in the interfacial plane. Thus, we cannot discuss further what kind of structures NPs form in the interfacial plane.

In any case, the reported $\phi$ (Fig. 5) could seem very small since one knows that desorption wetting energies $\Delta \mathrm{E}_{\mathrm{w}}$ are very high: ${ }^{35}$

$-\Delta \mathrm{E}_{\mathrm{W}}=\pi \mathrm{R}^{2} \gamma(1 \pm \cos \Theta)^{2}$, Eq. 9

where $\gamma$ is the interfacial tension, i.e. $\gamma=35 \mathrm{mN} \mathrm{m}^{-1}$ and $72 \mathrm{mN} \mathrm{m}^{-1}$ for water-toluene and waterair respectively. Plugging the experimental contact angle $\Theta$ values (Fig. 5) in the latter formula, for $\mathrm{NaOH}$ solution-air interface $\Delta \mathrm{E}_{\mathrm{w}}=-21 \mathrm{kT}$, for $\mathrm{NaCl}$ solution-air is $\Delta \mathrm{E}_{\mathrm{w}}=-1776 \mathrm{kT}$; and for 
aqueous solution-toluene is about $\Delta \mathrm{E}_{\mathrm{w}}=-2326 \mathrm{kT}$. Given that $\left|\Delta \mathrm{E}_{\mathrm{w}}\right|>>\mathrm{kT}$, particles should populate the interface at high $\phi$ values.

Here, it is worth noting that in the calculations of $\Delta \mathrm{E}_{\mathrm{w}}$ only the macroscopic wetting cost to remove a single particle from the interface to the bulk is evaluated and would be relevant for particles adsorbing from ideal suspensions onto ideal interfacial layers. In real systems, the adsorption equilibrium also depends on interparticle interactions, and the low surface coverage experimentally observed suggests strong repulsions at interface. In the Pieranski's model, in fact, the free energy profile of a particle with respect to its distance from the interface is calculated from both the interfacial energies of the particle and the planar interface. For particle desorption in the fluid $1, \Delta E_{w}=\left[\gamma_{1} A_{P}\right]-\left[\gamma_{1} A_{P, 1}+\gamma_{2} A_{P, 2}-\gamma A_{0}\right]$, where $\gamma_{1}, \gamma_{2}$ and $\gamma$ are the interfacial tension of particle-fluid 1, particle-fluid 2 and fluid 1- fluid 2 respectively; and $A_{P}, A_{P, 1}, A_{P, 2}$ and $A_{0}$ are the total area of the particle, particle area wetted by fluid 1, particle area wetted by fluid 2 and the area of the bare interface covered by the particle respectively. Thus, both the macroscopic fluid interface and the microscopic particle contribute to the total energy profile. ${ }^{35}$

Taking a different perspective, we could be tempted to discuss whether the surface coverages in the long time limit (plotted in Fig. 5) correspond to equilibrium concentration $\mathrm{c}_{\mathrm{S}}$ of an interphase, defined in a volume of thickness equal to the particle's diameter $2 \mathrm{R}_{1}, c_{S}=\frac{N \times 4 / 3 \pi R_{1}^{3}}{A \times 2 R_{1}}=\frac{2}{3} \phi$. In this microscopic description, the interfacial concentration is determined by the chemical potential equilibrium between bulk and the interfacial region, regarded as a real (inter)phase. ${ }^{36}$

Hence, we could write a partition coefficient between the interphase and bulk as $\mathrm{K}=\mathrm{c}_{\mathrm{S}} / \mathrm{c}_{\mathrm{NP}}=$ $\exp [-\Delta \mathrm{E} / \mathrm{kT}]$, where $\Delta \mathrm{E}$ is the difference in free energy between the interphase and the bulk. For aqueous-air interfaces, $\Delta \mathrm{E}=-6.6 \mathrm{kT}$; for $\mathrm{NaOH}$ aqueous-toluene interfaces $\Delta \mathrm{E}=-6.3 \mathrm{kT}$ and 
for $\mathrm{NaCl}$ aqueous-toluene interfaces $\Delta \mathrm{E}=-5.8 \mathrm{kT}$ can be calculated. Note that within this approach, a limiting $\Delta \mathrm{E}=-9.4 \mathrm{kT}$ is estimated for the fully packed value of $\phi=0.9$.

Now, the deepest energy minima at the interface are calculated for aqueous-air interfaces, on which only adsorption and not transfer (to air) can take place. NPs can cross the interface for $\mathrm{NaCl}$ aqueous solution-toluene interfaces, and in these cases the difference in free energy is also the smallest one, which confirms that the energetic states of bulk phases and the interphase are very close and a small thermodynamic change in one of the phase could lead to NP transfer.

Recent computer simulations have also shown that the NP energy profile across the interface cannot be described only accounting for wetting energies as in the Pieranski's model. ${ }^{35}$ As a matter of fact, the free energy of core-shell nanoparticles depends strongly on the grafting density and the hydrophobicity of the chemical species composing the particle's shell. ${ }^{37}$

4.3 Contact Angle. Finally, contact angle measurements (Fig. 5 and Table 1) can be discussed accounting for wetting and particle-interface interactions. In wetting, the Young-Laplace defines an equilibrium contact angle $\theta_{\mathrm{E}}$ from the far field equilibrium of the surface forces acting on the triple line, where the solid and the two fluids are in contact. ${ }^{3}$ In the vicinity of the triple line, as we go down in length scales, the far field equilibrium does not hold anymore and the local contact angle might deviate from the far field angle $\theta_{\mathrm{E}}$. At length scales smaller than $c a .30 \mathrm{~nm}$, detailed physicochemical properties of the solid and fluids affect the microscopic structure of the contact line; and van der Waals and electrostatic interactions modify the profile of the fluid line and the local contact angle. ${ }^{3}$

Hence, at least two equilibrium contact angles can be defined: a macroscopic contact angle that obeys to the Young-Laplace equation and a microscopic contact angle that depends strongly on colloidal interactions. 
The discussion so far is independent on the geometry of the wetting. Therefore, it applies to the case of a liquid drop on a solid substrate or to the wetting of a solid particle at the fluid interface. In the latter case, however, depending on its size, a solid particle may attain different equilibrium contact angles. The size of the particle is in fact a probing length scale of the wetting phenomenon. For micron-sized particles, the equilibrium contact angle attained by the particle is clearly the macroscopic one. ${ }^{38}$ Whereas, when the size of the particle is smaller than $c a .30 \mathrm{~nm}$, as in our case, one could expect to be in the microscopic regime.

In table 1, we compare macroscopic equilibrium contact angles with the ones obtained by ellipsometry in the long time limit. The latter measurements present the advantages of being noninvasive and to probe in situ the interfacial NP optical profile, which accounts for a large number of nanoparticles, and in turn evaluate microscopic contact angles. The main drawback of the method is that it relies on a model, which describes a monolayer of particles monodisperse both in size and in contact angle. To capture the contact angle distribution of polydisperse systems, other methods such as the freeze-fracture shadow-casting cryo-scanning electron microscopy are able to probe single nanoparticle wetting properties, even though they present the major drawback of being very invasive. ${ }^{11}$

We start discussing ellipsometric results obtained for aqueous-air interfaces, which show $\Theta=$ $67^{\circ} \pm 4^{\circ}$ for $\mathrm{NaCl}$ aqueous solution and $\Theta=21^{\circ} \pm 12^{\circ}$ for $\mathrm{NaOH}$ aqueous solution at $\mathrm{t}=6 \times 10^{5} \mathrm{~s}$. Note that for bare interfaces NPs do not adsorb onto the interface and $\Theta$ cannot be defined. The remarkable difference between the two contact angles supports the opposite effects of $\mathrm{NaCl}$ and $\mathrm{NaOH}$ in tuning the interaction of NPs and the aqueous medium. $\Theta(0.1 \mathrm{M} \mathrm{NaCl})>\Theta(0.1 \mathrm{M}$ $\mathrm{NaOH}$ ) corresponds to a lower affinity of $\mathrm{NP}$ for $\mathrm{NaCl}$ solution than for $\mathrm{NaOH}$ solution. 
Those results also agree with our previous investigations showing that $\mathrm{NaCl}$ promotes an hydrophobic character of the polymer shell, strongly shifting the lower critical solution temperature of the polymer in water. ${ }^{19}$

$\mathrm{NaOH}$ on the contrary not only promotes surface hydration of NPs but also increases the negative charge density at NP surface by deprotonation of the NP surface groups. The latter charge coupled with the expected negative charge of the water-air interface in turn might lead to a repulsion force, which push down to lower angles the NPs adsorbed onto the interface., ${ }^{4,6}$

For aqueous-air interfaces macroscopic equilibrium contact angles (table 1) do not show significant difference between $\mathrm{NaCl}$ and $\mathrm{NaOH}$ solutions, which is in agreement with the fact that in the far field colloidal interactions are not relevant. The only effect of the nature of the salt is to change the interfacial tensions of the aqueous interfaces.

A fairly good agreement between macroscopic equilibrium contact angles and in-situ contact angles by ellipsometry was found for water-oil interfaces.

For aqueous-toluene interfaces, NP contact angle is almost identical for $0.1 \mathrm{M} \mathrm{NaCl}$ and $\mathrm{NaOH}$ solutions, even though in the first case NPs cross the interface and transfer to toluene, whereas in the second case stay in the aqueous phase. Comparing to water-gas interfaces, charge effects are expected on both sides of the water-oil interface. Electrostatic and short-range interactions might lead to a steeper energetic landscape on both sides of the interface when compared to liquid-air interfaces and in the simple wetting case. This steep profile could be the reason why the contact angle remains close to $90^{\circ}$ for both $\mathrm{NaCl}$ and $\mathrm{NaOH}$ aqueous-toluene interfaces. A sketch of the energy landscapes for NPs at the fluid interfaces is shown in Fig. 6B.

\section{FIGURE 6}




\section{Conclusion}

In this article, we used ellipsometry to measure in-situ the contact angles and surface coverages of nanoparticles at the liquid-liquid and liquid-gas interfaces. Gold nanoparticles adsorb onto the fluid interface only when salt is present in the system, pointing to the existence of a potential barrier close to the interface. In the long time limit, we discussed equilibrium or pseudo equilibrium surface coverages and contact angles under the hypothesis of equilibrium between the bulk phases and the inter(phase). For aqueous-air interfaces, nanoparticle contact angles depends strongly on the nature of the salt, whereas at aqueous-toluene interfaces the in-situ contact angles are almost identical for both $\mathrm{NaCl}$ or $\mathrm{NaOH}$ solutions. The energy landscape of NPs at the interface is rather complex: wetting, electrostatic and physicochemical properties of water interfaces affect the NP contact angles, which could become different from the macroscopic contact angles measured in a wetting experiment.

These results are particularly relevant for NP phase transfer, and for emulsions and foams stabilized by NPs. Measuring in-situ both the contact angle and the surface coverage is in fact crucial in order to understand and model NP transfer and emulsion/foam stability.

The present method is of particular interest to contribute bridging the long lasting gap between the different scales that are relevant in wetting phenomena. Its advantages rely on measurement over large ensembles of particles, the probing scale of which being defined by the size of the nanoparticles used. In the future, we plan systematic investigations as a function of salt and nanoparticle concentrations in order to elucidate on interfacial interactions and adsorption isotherm of nanoparticle at the fluid interface. 


\section{Acknowledgment.}

The authors are grateful to Helmuth Möhwald and Reinhard Miller for their contributions in the

discussion and for encouraging and supporting this work. A. S. acknowledges financial support from Labex Numev (ANR-10-LABX-20) and Labex CheMISyst. 
1. N. Belman, K. Jin, Y. Golan, J. N. Israelachvili, and N. S. Pesika, Langmuir, 2012, 28, 14609-17.

2. B. P. Binks and R. Murakami, Nat. Mater., 2006, 5, 865-9.

3. P.-G. De Gennes, Rev. Mod. Phys., 1985, 57, 827-863.

4. T. Gehring and T. M. Fischer, J. Phys. Chem. C, 2011, 115, 23677-23681.

5. T. M. Fischer, P. Dhar, and P. Heinig, J. Fluid Mech., 2006, 558, 451.

6. A. Shrestha, K. Bohinc, and S. May, Langmuir, 2012, 28, 14301-7.

7. S. Sacanna, W. Kegel, and a. Philipse, Phys. Rev. Lett., 2007, 98, 13-16.

8. A. S. Almusallam and D. S. Sholl, J. Colloid Interface Sci., 2007, 313, 345-52.

9. A. S. Almusallam, Phys. Chem. Chem. Phys., 2008, 10, 3099-107.

10. a Maestro, L. J. Bonales, H. Ritacco, R. G. Rubio, and F. Ortega, Phys. Chem. Chem. Phys., 2010, 12, 14115-20.

11. L. Isa, F. Lucas, R. Wepf, and E. Reimhult, Nat. Commun., 2011, 2, 438.

12. L. Isa, D. C. E. Calzolari, D. Pontoni, T. Gillich, A. Nelson, R. Zirbs, A. Sánchez-Ferrer, R. Mezzenga, and E. Reimhult, Soft Matter, 2013, 9, 3789.

13. D. C. E. Calzolari, D. Pontoni, M. Deutsch, H. Reichert, and J. Daillant, Soft Matter, 2012, 8, 11478 .

14. G. Kaptay, Colloids Surfaces A Physicochem. Eng. Asp., 2003, 230, 67-80.

15. J. C. H. Wong, E. Tervoort, S. Busato, U. T. Gonzenbach, A. R. Studart, P. Ermanni, J. Ludwig, and L. J. Gauckler, J. Mater. Chem., 2009, 19, 5129.

16. T. N. Hunter, G. J. Jameson, and E. J. Wanless, Aust. J. Chem., 2007, 60, 651.

17. D. Zang, A. Stocco, D. Langevin, B. Wei, and B. P. Binks, Phys. Chem. Chem. Phys., 2009, 11, 9522-9.

18. A. Stocco, T. Mokhtari, G. Haseloff, A. Erbe, and R. Sigel, Phys. Rev. E, 2011, 83, 1-11.

19. A. Stocco, M. Chanana, G. Su, P. Cernoch, B. P. Binks, and D. Wang, Angew. Chemie Int. Ed., 2012, 51, 9647-51. 
20. E. W. Edwards, M. Chanana, D. Wang, and H. Möhwald, Angew. Chemie, 2008, 120, 326-329.

21. R. L. Olmon, B. Slovick, T. W. Johnson, D. Shelton, S.-H. Oh, G. D. Boreman, and M. B. Raschke, Phys. Rev. B, 2012, 86, 235147.

22. P. Stoller, V. Jacobsen, and V. Sandoghdar, Opt. Lett., 2006, 31, 2474-2476.

23. W. Haiss, N. T. K. Thanh, J. Aveyard, and D. G. Fernig, Anal. Chem., 2007, 79, 4215-21.

24. R. M. Azzam and N. M. Bashara, Ellipsometry and polarized light, North-Holland. sole distributors for the USA and Canada, Elsevier Science Publishing Co., Inc., 1987.

25. J. Lekner, Theory of Reflection, 1987.

26. R. Tadmor, Langmuir, 2004, 20, 7659-64.

27. J. Day and C. Bain, Phys. Rev. E, 2007, 76, 041601.

28. A. Stocco and K. Tauer, Eur. Phys. J. E. Soft Matter, 2009, 30, 431-8.

29. C. Stefaniu, M. Chanana, H. Ahrens, D. Wang, G. Brezesinski, and H. Möhwald, Soft Matter, 2011, 7, 4267.

30. V. Garbin, J. C. Crocker, and K. J. Stebe, J. Colloid Interface Sci., 2012, 387, 1-11.

31. M. Oettel and S. Dietrich, Langmuir, 2008, 24, 1425-41.

32. E. W. Edwards, M. Chanana, and D. Wang, J. Phys. Chem. C, 2008, 112, 15207-15219.

33. F. Ravera, M. Ferrari, E. Santini, and L. Liggieri, Adv. Colloid Interface Sci., 2005, 117, 75-100.

34. R. Aveyard, J. H. Clint, D. Nees, and V. N. Paunov, Langmuir, 2000, 1969-1979.

35. P. Pieranski, Phys. Rev. Lett., 1980, 45, 569.

36. H. Brenner and L. . Leal, J. Colloid Interface Sci., 1978, 65, 191-209.

37. R. J. K. Udayana Ranatunga, R. J. B. Kalescky, C. Chiu, and S. O. Nielsen, J. Phys. Chem. $C, 2010,114,12151-12157$.

38. C. Blanc, D. Fedorenko, M. Gross, M. In, M. Abkarian, M. A. Gharbi, J.-B. Fournier, P. Galatola, and M. Nobili, Phys. Rev. Lett., 2013, 111, 058302. 
Table 1. Macroscopic static $\left(\theta_{\mathrm{S}}\right)$ advancing $\left(\theta_{\mathrm{A}}\right)$ receding $\left(\theta_{\mathrm{R}}\right)$ and equilibrium $\left(\theta_{\mathrm{E}}\right)^{26}$ contact angle measurements on Au@MEOMA90-co-OEGMA10 model wafer (captive bubble/drop method). For comparison, contact angle $\Theta$ measurements by ellipsometry in the long time limit are also shown.

\begin{tabular}{|l|l|l|l|l|l|l|}
\hline & $\begin{array}{l}\text { water in } \\
\text { air }\end{array}$ & $\begin{array}{l}0.1 \mathrm{M} \mathrm{NaCl} \\
\text { in air }\end{array}$ & $\begin{array}{l}0.1 \mathrm{M} \mathrm{NaOH} \\
\text { in air }\end{array}$ & $\begin{array}{l}\text { water in } \\
\text { toluene }\end{array}$ & $\begin{array}{l}0.1 \mathrm{M} \mathrm{NaCl} \\
\text { in toluene }\end{array}$ & $\begin{array}{l}0.1 \mathrm{M} \mathrm{NaOH} \\
\text { in toluene }\end{array}$ \\
\hline$\theta_{\mathrm{S}}$ & $61.8 \pm 0.8$ & $62.6 \pm 0.7$ & $59.3 \pm 1.2$ & $105.3 \pm 0.3$ & $104.4 \pm 0.3$ & $107.2 \pm 2.5$ \\
\hline$\theta_{\mathrm{A}}$ & $66.6 \pm 1.3$ & $64.4 \pm 0.8$ & $66.6 \pm 0.7$ & $108.9 \pm 0.7$ & $108.3 \pm 0.9$ & $109.0 \pm 0.5$ \\
\hline$\theta_{\mathrm{R}}$ & $40.7 \pm 0.6$ & $40.6 \pm 0.7$ & $40.3 \pm 1.8$ & $81.0 \pm 1.4$ & $78.8 \pm 1.8$ & $72.0 \pm 1.5$ \\
\hline$\theta_{\mathrm{E}}$ & $53.3 \pm 0.9$ & $\mathbf{5 2 . 2} \pm \mathbf{0 . 7}$ & $\mathbf{5 3 . 1} \pm \mathbf{1 . 2}$ & $93.0 \pm 1.1$ & $\mathbf{9 1 . 5} \pm \mathbf{1 . 4}$ & $\mathbf{8 7 . 4 \pm 1 . 0}$ \\
\hline$\Theta$ & & $\mathbf{6 7 . 5} \pm \mathbf{4 . 6}$ & $\mathbf{2 1 . 5} \pm \mathbf{1 2 . 2}$ & & $\mathbf{9 1 . 3} \pm \mathbf{0 . 4}$ & $\mathbf{9 0 . 0} \pm \mathbf{0 . 6}$ \\
\hline
\end{tabular}



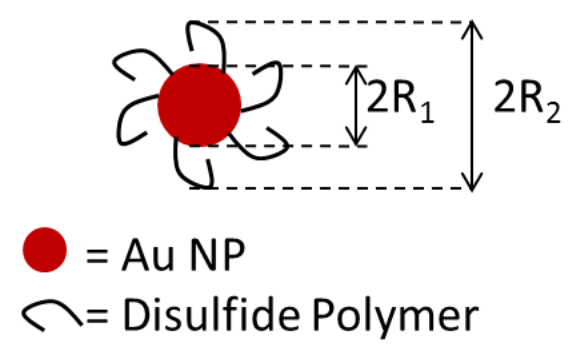

\section{A}
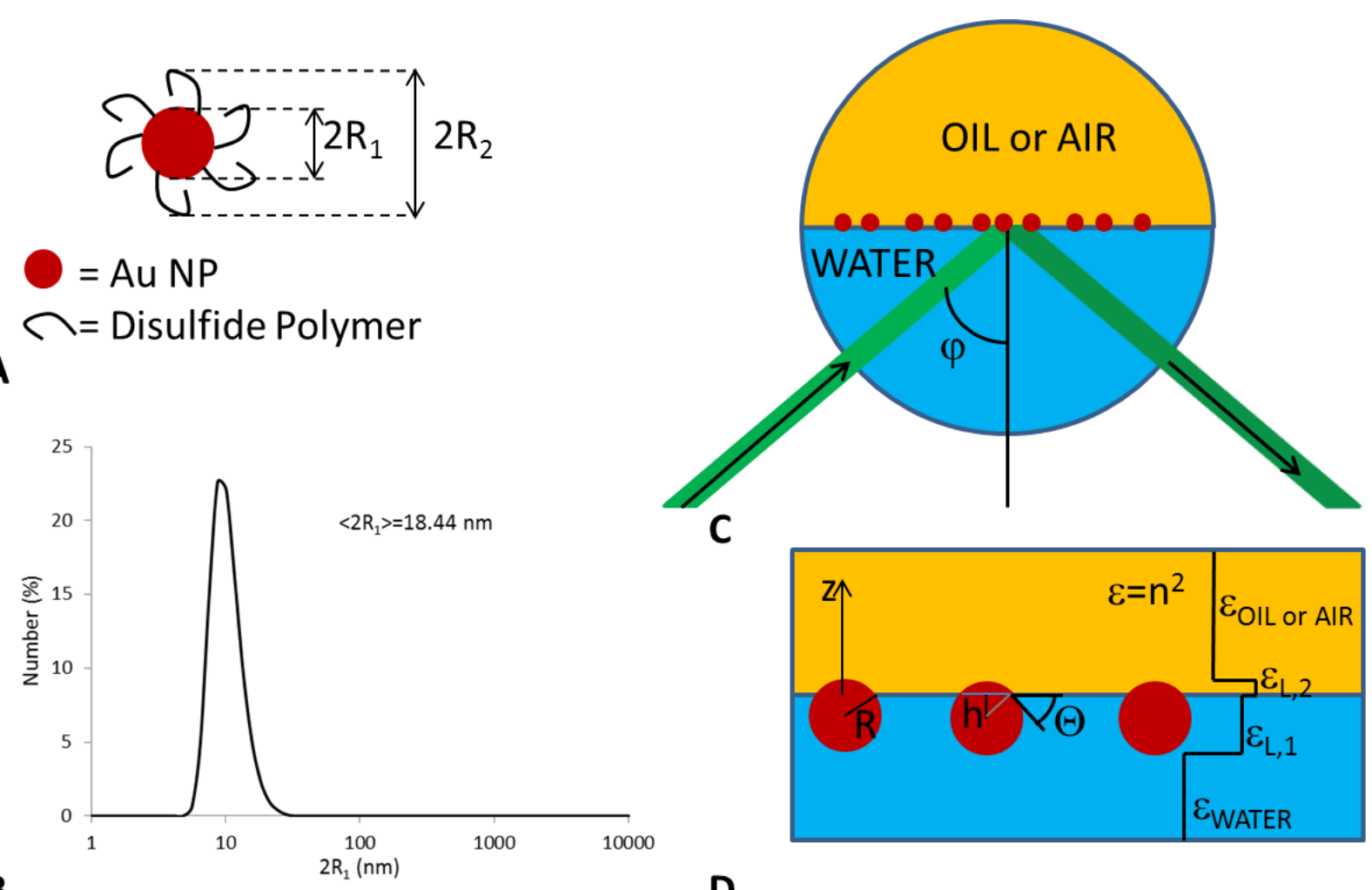

B

D

Figure 1

(A) Sketch of the core-shell NP and corresponding radii. (B) Number distribution of necked gold NPs obtained by dynamic light scattering. (C) Sketch of the ellipsometric measurement scheme with the laser incident from the bottom of a water-fluid interface contained in a cylindrical cell sample. (D) Sketch of the nanoparticle ellipsometric model: $\mathrm{R}$ is the NP radius, $\mathrm{h}$ is the distance between the NP's center and the interface ( $h<0$ in the sketch), see the text for details. 

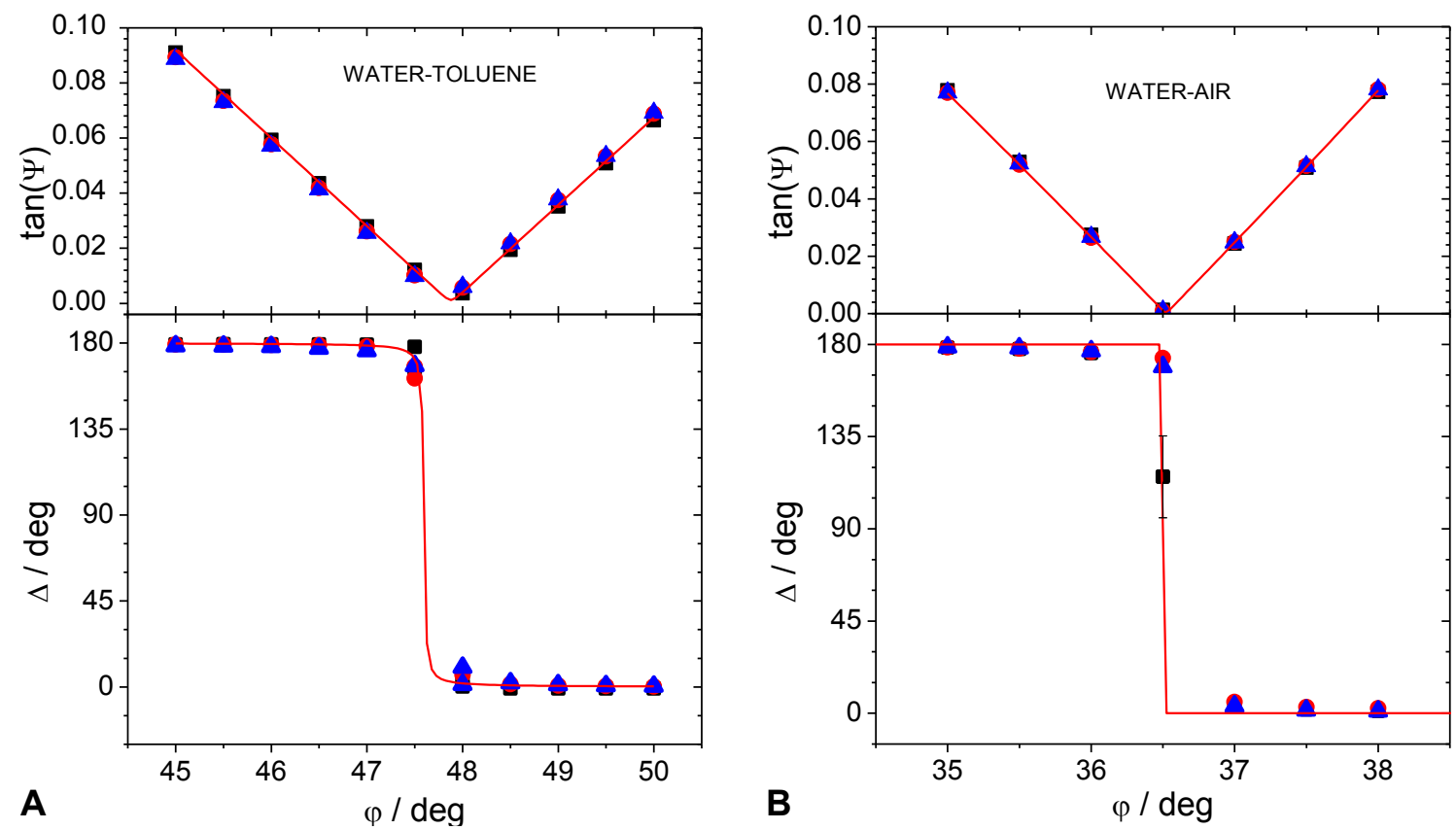

Figure 2

Measurements of $\tan \Psi$ and $\Delta$ as a function of the incident angle for water-toluene (A) and waterair (B) interfaces: bare interfaces ( $\mathbf{\square}$ ) and aqueous dispersion of gold NPs c $=1 \mathrm{gL}^{-1}$ measured at adsorption times $t=7200 \mathrm{~s}(\mathbf{O})$ and $86400 \mathrm{~s}(\boldsymbol{\Delta})$. Solid lines represent fits according to the perturbation theory. 

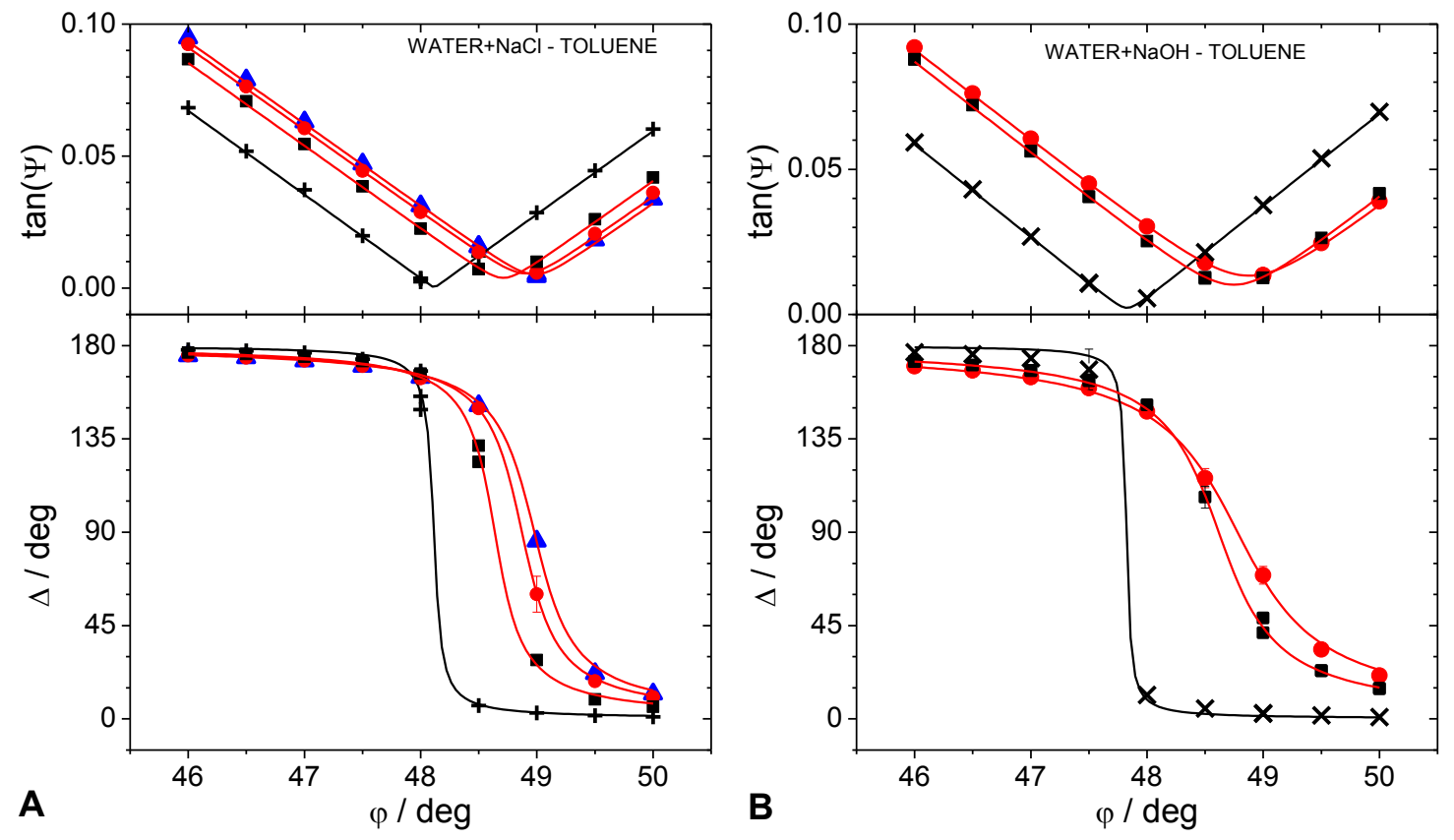

Figure 3: Aqueous solution-toluene interface:

(A) Measurements of $\tan \Psi$ and $\Delta$ as a function of the incident angle for $0.1 \mathrm{M} \mathrm{NaCl}$ aqueous solution-toluene interfaces before $(+)$ and after the adsorption of gold NPs measured at $t=10^{5} \mathrm{~s}$ (ם), $1.8 \times 10^{5} \mathrm{~s}(\mathbf{O})$ and $4.3 \times 10^{5} \mathrm{~s}(\boldsymbol{\Delta})$.

(B) Measurements of $\tan \Psi$ and $\Delta$ as a function of the incident angle for $0.1 \mathrm{M} \mathrm{NaOH}$ aqueous solution-toluene interfaces before $(\mathbf{X})$ and after the adsorption of gold NPs measured at $\mathrm{t}=10^{5} \mathrm{~s}$ (ם) and $6.1 \times 10^{5} \mathrm{~s}(\bigcirc)$. Black lines represent fits according to the perturbation theory to the data. Red lines represent fits according to the nanoparticle layer model. 

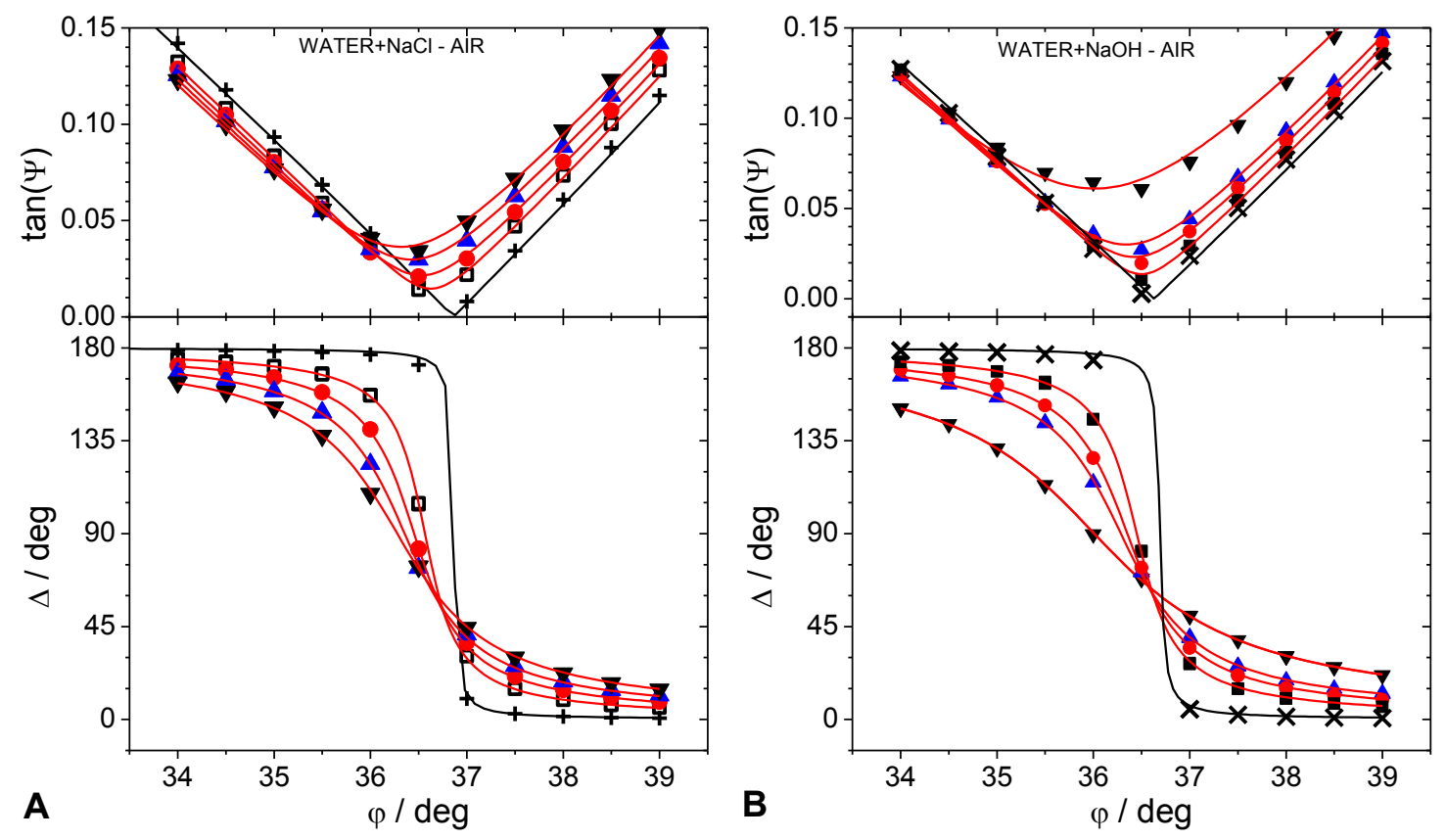

Figure 4: Aqueous solution-air interface:

(A) Measurements of $\tan \Psi$ and $\Delta$ as a function of the incident angle for $0.1 \mathrm{M} \mathrm{NaCl}$ aqueous solution-toluene interfaces before $(+)$ and after the adsorption of gold NPs measured at $\mathrm{t}=$ $0.9 \times 10^{5} \mathrm{~s}(\boldsymbol{\square}), 1.8 \times 10^{5} \mathrm{~s}(\mathbf{O}), 2.6 \times 10^{5} \mathrm{~s}(\boldsymbol{\Delta})$ and $6 \times 10^{5} \mathrm{~s}(\boldsymbol{\nabla})$.

(B) Measurements of $\tan \Psi$ and $\Delta$ as a function of the incident angle for $0.1 \mathrm{M} \mathrm{NaOH}$ aqueous solution-toluene interfaces before $(\mathbf{X})$ and after the adsorption of gold NPs measured at $\mathrm{t}=$ $0.9 \times 10^{5} \mathrm{~s}(\boldsymbol{\square}), 1.8 \times 10^{5} \mathrm{~s}(\mathbf{O}), 2.6 \times 10^{5} \mathrm{~s}(\boldsymbol{\Delta})$ and $6 \times 10^{5} \mathrm{~s}(\boldsymbol{\nabla})$.

Black lines represent fits of the perturbation theory to the data. Red lines represent fits of the nanoparticle ellipsometric model to the data. 

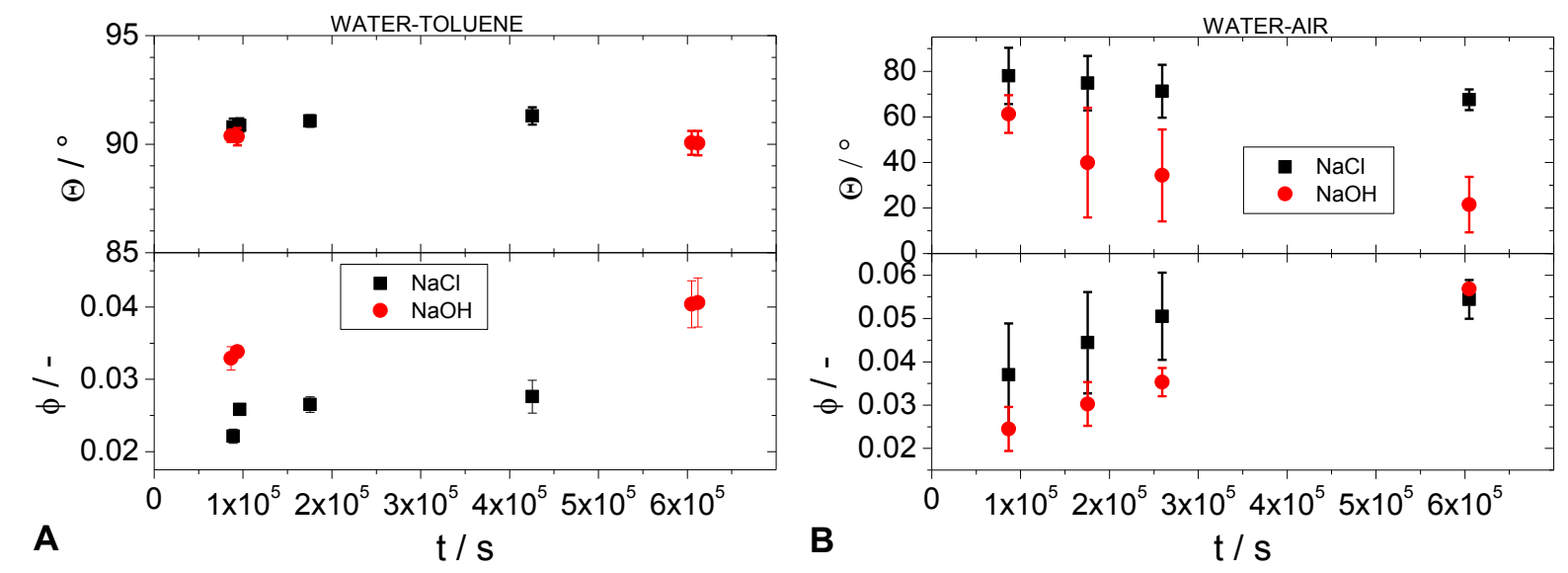

Figure 5

Contact angle $\Theta$ and surface coverage $\phi$ as a function of adsorption time for aqueous-toluene (A) and aqueous-air (B) interfaces in presence of $0.1 \mathrm{M} \mathrm{NaCl}(\mathbf{\square})$ and $\mathrm{NaOH}(\mathbf{O})$ obtained from the fits of the nanoparticle ellipsometric model to the data. 


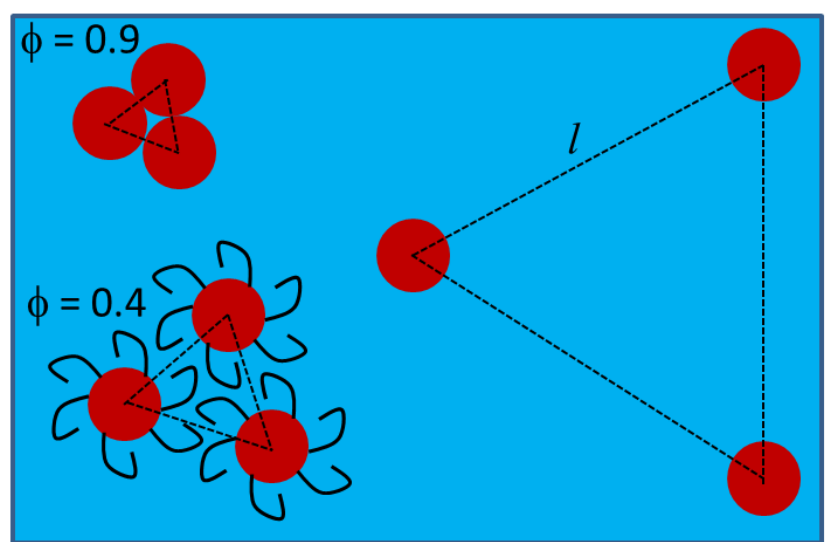

A

Figure 6

(A) NP packing at the water surface for $\phi=0.9,0.4$ and the general case where the distance between particle's centers is $l$. (B) Interfacial free energy profiles at the aqueous-toluene and aqueous-air interfaces. The dashed profile corresponds to pure water, the grey profiles to $\mathrm{NaOH}$ aqueous solution and the solid black profiles to $\mathrm{NaCl}$ aqueous solutions. 
APPENDIX. Core-shell NP ellipsometric model.

In the core-shell model, a spherical particle's geometry is assumed, in which a radius of the core $R_{C O R E}$ and one of the entire core-shell particle $R_{C S}\left(R_{C S}>R_{C}\right)$ can be defined (see Fig. A1). The surface coverage is now:

$\phi=\mathrm{N} \pi \mathrm{R}_{\mathrm{CS}}{ }^{2} / \mathrm{A}$.

The thickness of the first layer is $\mathrm{R}_{\mathrm{CS}}-\mathrm{h}$ and the one of the second layer is $\mathrm{R}_{\mathrm{CS}}+\mathrm{h}$; and the dielectric constants of the two layers are:

$$
\begin{aligned}
& \varepsilon_{L 1}=\varepsilon_{1} \frac{\varepsilon_{1}\left(2-2 f_{C S N P, 1}\right)+\varepsilon_{C S N P, 1}\left(1+2 f_{C S N P, 1}\right)}{\varepsilon_{1}\left(2+f_{C S N P, 1}\right)+\varepsilon_{C S N P, 1}\left(1-f_{C S N P, 1}\right)} \\
& \varepsilon_{L 2}=\varepsilon_{2} \frac{\varepsilon_{2}\left(2-2 f_{C S N P, 2}\right)+\varepsilon_{C S N P, 2}\left(1+2 f_{C S N P, 2}\right)}{\varepsilon_{2}\left(2+f_{C S N P, 2}\right)+\varepsilon_{C S N P, 2}\left(1-f_{C S N P, 2}\right)}
\end{aligned}
$$

$\varepsilon_{\mathrm{CSNP}, 1}$ and $\varepsilon_{\mathrm{CSNP}, 2}$ are the dielectric constants of core-shell nanoparticles. $f_{C S N P, 1}$ and $f_{C S N P, 1}$ are the volume fractions of the portion of core-shell particles immersed in the layer 1 and 2 respectively:

$$
f_{C S N P, 1}=\phi \frac{2 / 3 R_{C S}{ }^{3}-\left(R_{C S}{ }^{2} h-h^{3} / 3\right)}{R_{C S}{ }^{2}\left(R_{C S}-h\right)}, f_{C S N P, 2}=\phi \frac{2 / 3 R_{C S}{ }^{3}+\left(R_{C S}{ }^{2} h-h^{3} / 3\right)}{R_{C S}{ }^{2}\left(R_{C S}+h\right)} .
$$

Hence, we used the Wiener effective medium approximation to calculate $\varepsilon_{\mathrm{CSNP}, 1}$ and $\varepsilon_{\mathrm{CSNP}, 2}$ :

$$
\varepsilon_{C S N P, 1}=f_{C O R E, 1} \varepsilon_{C O R E}+\left(1-f_{C O R E, 1}\right) \varepsilon_{S H, 1}, \varepsilon_{C S N P, 2}=f_{C O R E, 2} \varepsilon_{C O R E}+\left(1-f_{C O R E, 2}\right) \varepsilon_{S H, 2} . \quad \text { Eq. A5 }
$$

Where $\varepsilon_{\mathrm{SH}, 1}$ and $\varepsilon_{\mathrm{SH}, 2}$ are the dielectric constants of the particle shell in layer 1 and 2 respectively (see Fig. A1).

$$
f_{C O R E, 1}=\frac{2 / 3 R_{\text {CORE }}{ }^{3}-\left(R_{C O R E}{ }^{2} h-h^{3} / 3\right)}{2 / 3 R_{C S}{ }^{3}-\left(R_{C S}{ }^{2} h-h^{3} / 3\right)}, f_{C O R E, 2}=\frac{2 / 3 R_{C O R E}{ }^{3}+\left(R_{C O R E}{ }^{2} h-h^{3} / 3\right)}{2 / 3 R_{C S}{ }^{3}+\left(R_{C S}{ }^{2} h-h^{3} / 3\right)}, \quad \text { Eq. A6 }
$$


are the fractions of the volume occupied by the core with respect to the whole core-shell particles. Finally, when the particle's shell is composed by polymer and solvent, the refractive index changes linearly with the polymer volume fractions $f_{\mathrm{POL}}$ :

$\varepsilon_{S H, 1}=n_{S H, 1}^{2}$ and $n_{S H, 1}=f_{P O L} n_{P O L}+\left(1-f_{P O L}\right) n_{1}$

$\varepsilon_{S H, 2}=n_{S H, 2}^{2}$ and $n_{S H, 2}=f_{P O L} n_{P O L}+\left(1-f_{P O L}\right) n_{2}$

where $\mathrm{n}_{\mathrm{POL}}$ is the bulk refractive index of the polymer.

Note that when $\mathrm{h}>\mathrm{R}_{\mathrm{CORE}}, f_{\mathrm{CORE}, 1}=0$ and $f_{\text {CORE }, 2}=\frac{4 / 3 R_{\text {CORE }}{ }^{3}}{2 / 3 R_{C S}{ }^{3}+\left(R_{C S}{ }^{2} h-h^{3} / 3\right)}$;

and if $\mathrm{h}<-\mathrm{R}_{\mathrm{CORE}} f_{\mathrm{CORE}, 1}=\frac{4 / 3 R_{\text {CORE }}{ }^{3}}{2 / 3 R_{C S}{ }^{3}-\left({R_{C S}}^{2} h-h^{3} / 3\right)}$ and $f_{\mathrm{CORE}, 2}=0$.

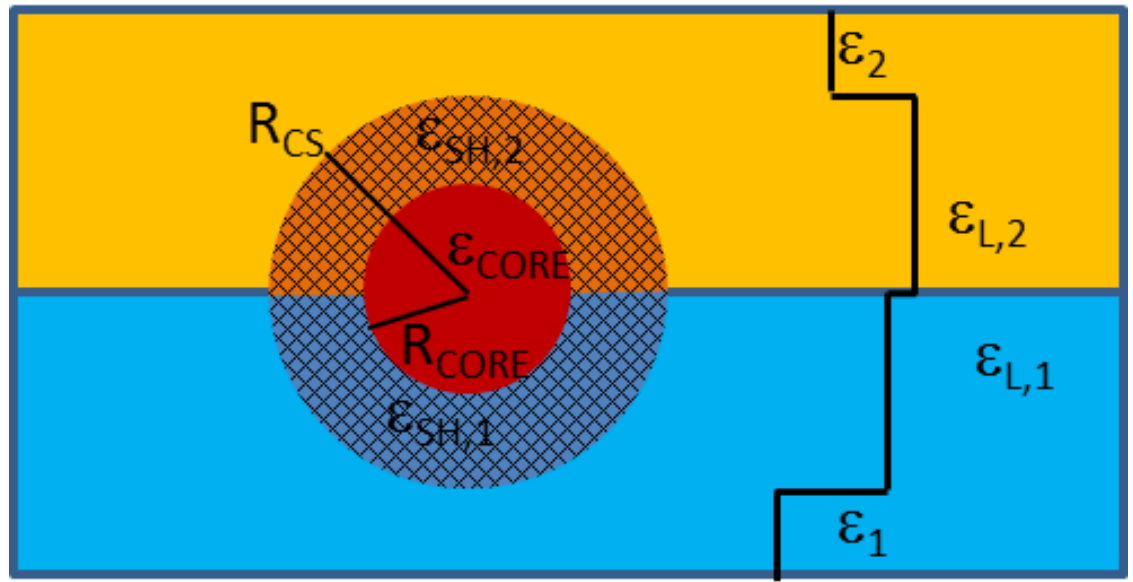

Fig. A1. Sketch of core-shell NP ellipsometric model. 